\title{
Impedance of a Monopole Antenna With a Radial-Wire Ground System on an Imperfectly Conducting Half Space, Part I
}

\author{
S. W. Maley and R. J. King
}

\author{
Contribution from Electrical Engineering Dept., University of Colorado, Boulder, Colo., supported by \\ Electronics Directorate of USAF Cambridge Research Center
}

(Received October 5, 1961)

\begin{abstract}
The effectiveness of a radial wire ground system as an approximation to a radial conducting disk ground system for a vertical monopole antenna over an imperfectly conducting ground is investigated experimentally by means of impedance measurements. The results were compared with theoretical work by J. R. Wait. The comparison shows that Wait's formula for the effective surface impedance of a radial wire ground system gives results which agree well with the measurements.
\end{abstract}

\section{Introduction}

A vertical monopole antenna over an imperfectly conducting ground is normally used in conjunction with a ground system consisting of a circular conducting disk or a number of radial wires. The use of such a ground system greatly reduces the power loss in the ground near the base of the monopole. The disk is the most effective ground system in this respect; but, for large antenna systems, cost considerations necessitate the use of radial wires. The effectiveness of radial wire systems as approximations to disks is investigated by means of impedance measurements.

A vertical monopole antenna system was constructed in the laboratory using water as an imperfectly conducting ground. The water was contained in a tank of sufficient extent and depth to very closely approximate an infinite half space.

Impedance measurements were made for a $\lambda / 4$ monopole, first with a circular conducting disk ground system and then with radial wire ground systems of various radii and number of wires. The frequencies used were 4.2 and $9.6 \mathrm{kMc} / \mathrm{s}$. As the number of radial wires in the ground system is increased, the impedance of the monopole approaches that for a conducting disk ground system of equal radius. The impedance as a function of the number of radial wires is plotted on the same charts with calculated impedince values based on a formula by Wait [1954].

\section{Impedance Calculations}

The impedance of a monopole antenna over an imperfectly conducting ground is given by Wait $[1954]$ as $^{1}$

$$
Z=Z^{\infty}-\frac{2 \pi}{I_{0}^{2}} \int_{0}^{\infty} H_{\phi}^{\infty}(r, 0) E_{r}(r, 0) r d r .
$$

$Z^{\infty}$ and $H_{\phi}^{\infty}(r, 0)$ are quantities which would exist if the ground were perfectly conducting. $Z^{\infty}$ is the monopole base impedance, and $H_{\phi}^{\infty}(r, 0)$ is the magnetic field at the surface of the ground for a monopole base current of $I_{0} . \quad E_{r}(r, 0)$ is the tangential component of the electric field which exists for a monopole base current of $I_{0}$ and for the imperfectly conducting ground. The time dependence is $\exp (i \omega t)$ where $\omega$ is angular frequency, $t$ is time and $i=\sqrt{-1}$. The coordinate system is shown in figure 1.

For the case of a conducting disk ground system of radius " $a$," $E_{r}(r, 0)=0$ for $0<r<a$ and (1) becomes

$$
Z=Z^{\infty}-\frac{2 \pi}{I_{0}^{2}} \int_{a}^{\infty} H_{\phi}^{\infty}(r, 0) E_{r}(r, 0) r d r .
$$

For the case of a radial wire ground system. such as shown in figure 2 the wires are connected to a small metal disk of radius " $a$ "; therefore, (1) becomes

$$
\begin{aligned}
Z=Z^{\infty}-\frac{2 \pi}{I_{0}^{2}} \int_{a}^{b} H_{\phi}^{\infty}(r, 0) & E_{r}(r, 0) r d r \\
& -\frac{2 \pi}{I_{0}^{2}} \int_{b}^{\infty} H_{\phi}^{\infty}(r, 0) E_{r}(r, 0) r d r
\end{aligned}
$$

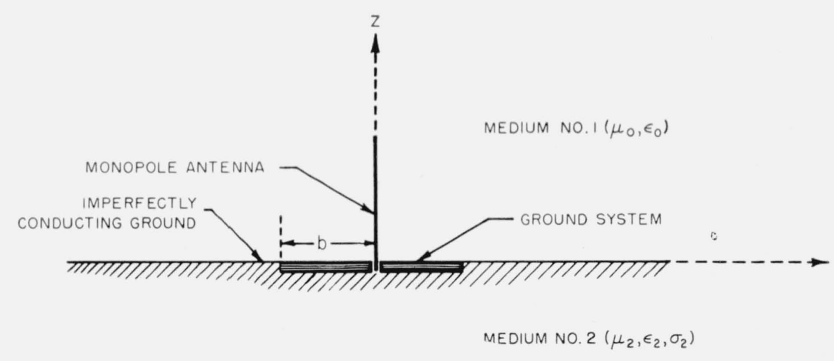

Figure 1. Monopole antenna and ground system on the surface of an imperfectly conducting half space.

The rationalized MKS system of units is used for all equations. 


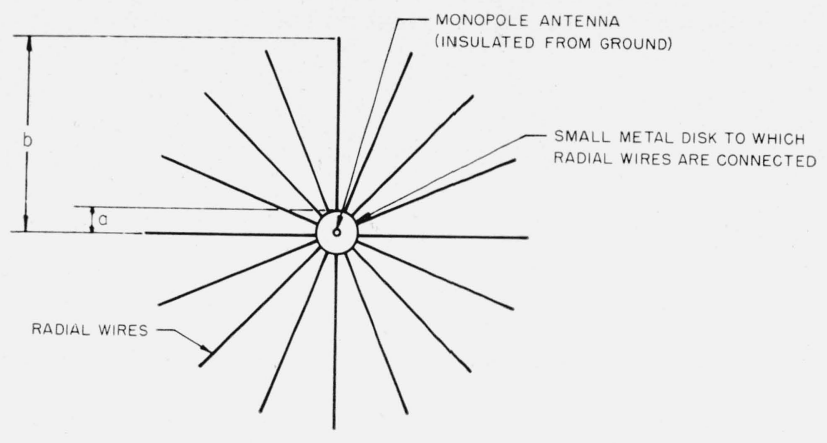

Figure 2, Top view of a radial wire ground system.

$E_{r}(r, 0)$ in the two integrals of $(3)$ is the electric field with the radial-wire ground system in place.

The assumption is now made that the boundary at the surface of the ground can be approximated by an impedance boundary; so we have the approximate relation,

$$
E_{r}(r, 0) \cong-\eta H_{\phi}(r, 0) \cong-\eta H_{\phi}^{\infty}(r, 0),
$$

where the surface impedance, $\eta$, can be a constant or a function of position, depending upon the ground system. This approximation is valid if $|\eta|$ is sufficiently small [Wait, 1954].

Using (4), (2) and (3) now become

$$
Z \cong Z^{\infty}+\frac{2 \pi}{I_{0}^{2}} \int_{a}^{\infty} \eta_{g}\left[H_{\phi}^{\infty}(r, 0)\right]^{2} r d r
$$

and

$$
\begin{aligned}
Z \cong Z^{\infty}+\frac{2 \pi}{I_{0}^{2}} \int_{a}^{b} \eta_{e}\left[H_{\phi}^{\infty}(r, 0)\right]^{2} r d r & \\
& +\frac{2 \pi}{I_{0}^{2}} \int_{b}^{\infty} \eta_{g}^{\prime}\left[H_{\phi}^{\infty}(r, 0)\right]^{2} r d r .
\end{aligned}
$$

$\eta_{g}$ is the surface impedance of the ground without a radial-wire system. $\eta_{g}^{\prime}$ and $\eta_{e}$ are the surface impedances of the ground for $b<r$ and $a<r<b$ respectively with the radial-wire system in place.

$\eta_{g}^{\prime}$ may be approximated to a good order of accuracy by $\eta_{g}$ [Wait, 1954; Maley, King, 1961]; thus

$$
\eta_{g}^{\prime} \cong \eta_{g}=\left[\frac{i \mu_{2} \omega}{\sigma_{2}+i \omega \epsilon_{2}}\right]^{\frac{1}{2}}
$$

The impedance, $\eta_{w}$, of a radial-wire system in free space has been given by Wait [1954] as

$$
\eta_{w}=\frac{i 2 \pi \eta_{0} r}{\lambda N} \ln \frac{r}{N c}
$$

where $\lambda$ is the free space wavelength, $N$ is the number of radial wires, and $c$ is the radius of the wires.

Since the radial-wire system is on the surface of the ground, it seems reasonable to place the impedances $\eta_{g}$ and $\eta_{w}$ in parallel to give $\eta_{e}$; thus [Wait, $1954]$

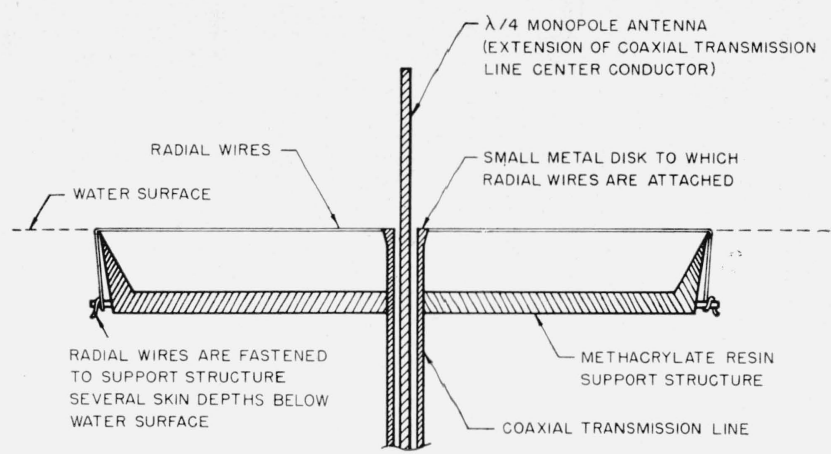

FIGURE 3. Radial wire ground system used for impedance measurements.

$$
\eta_{e}=\frac{\eta_{g} \eta_{w}}{\eta_{g}+\eta_{w}}
$$

This procedure has recently been given strong justification [Wait, 1959].

We now need expressions for $Z^{\infty}$ and $H_{\phi}^{\infty}(r, 0)$. These can be calculated by assuming a current distribution on the antenna; however, these quantities can also be obtained from measurements of the impedance of the monopole with a circular conducting disk ground system. The impedance of the monopole with the disk ground system on the surface of the water was measured as a function of disk radius " $a$," and is plotted in figure 4 for a frequency of 4.2 k.Mc/s. $\quad Z^{\infty}$ can be obtained by extrapolation from these curves [Maley, King, 1961; Johnk, 1960]. Differentiation of (5) with respect to " $a$ " gives

$$
\frac{d Z}{d a} \cong-\frac{2 \pi}{I_{0}^{2}} \eta_{g}\left[H_{\phi}^{\infty}(r, 0)\right]^{2} r .
$$

Using (7), the quantity

$$
\frac{2 \pi}{I_{\mathrm{d}}^{2}}\left[H_{\phi}^{\infty}(r, 0)\right]^{2} r
$$

can therefore be evaluated in terms of the slope of the curves of $Z$ as a function of " $a$ " shown in figure 4.

Equations (6), (7), (8), (9), (10), and figure 4 may now be used to calculate the impedance for the monopole with the radial-wire ground system. The effect of the number and length of the radial wires is of primary concern in this part of the investigation; therefore, from (6) we may obtain the expression

$$
\begin{aligned}
Z & -Z^{\infty}-\frac{2 \pi}{I_{0}^{2}} \int_{a}^{\infty} \eta_{g}\left[H_{\phi}^{\infty}(r, 0)\right]^{2} r d r \\
= & \frac{2 \pi}{I_{0}^{2}} \int_{a}^{b} \eta_{e}\left[H_{\phi}^{\infty}(r, 0)\right]^{2} r d r+\frac{2 \pi}{I_{0}^{2}} \int_{b}^{\infty} \eta_{g}^{\prime}\left[H_{\phi}^{\infty}(r, 0)\right]^{2} r d r \\
& -\frac{2 \pi}{I_{0}^{2}} \int_{a}^{\infty} \eta_{g}\left[H_{\phi}^{\infty}(r, 0)\right]^{2} r d r
\end{aligned}
$$



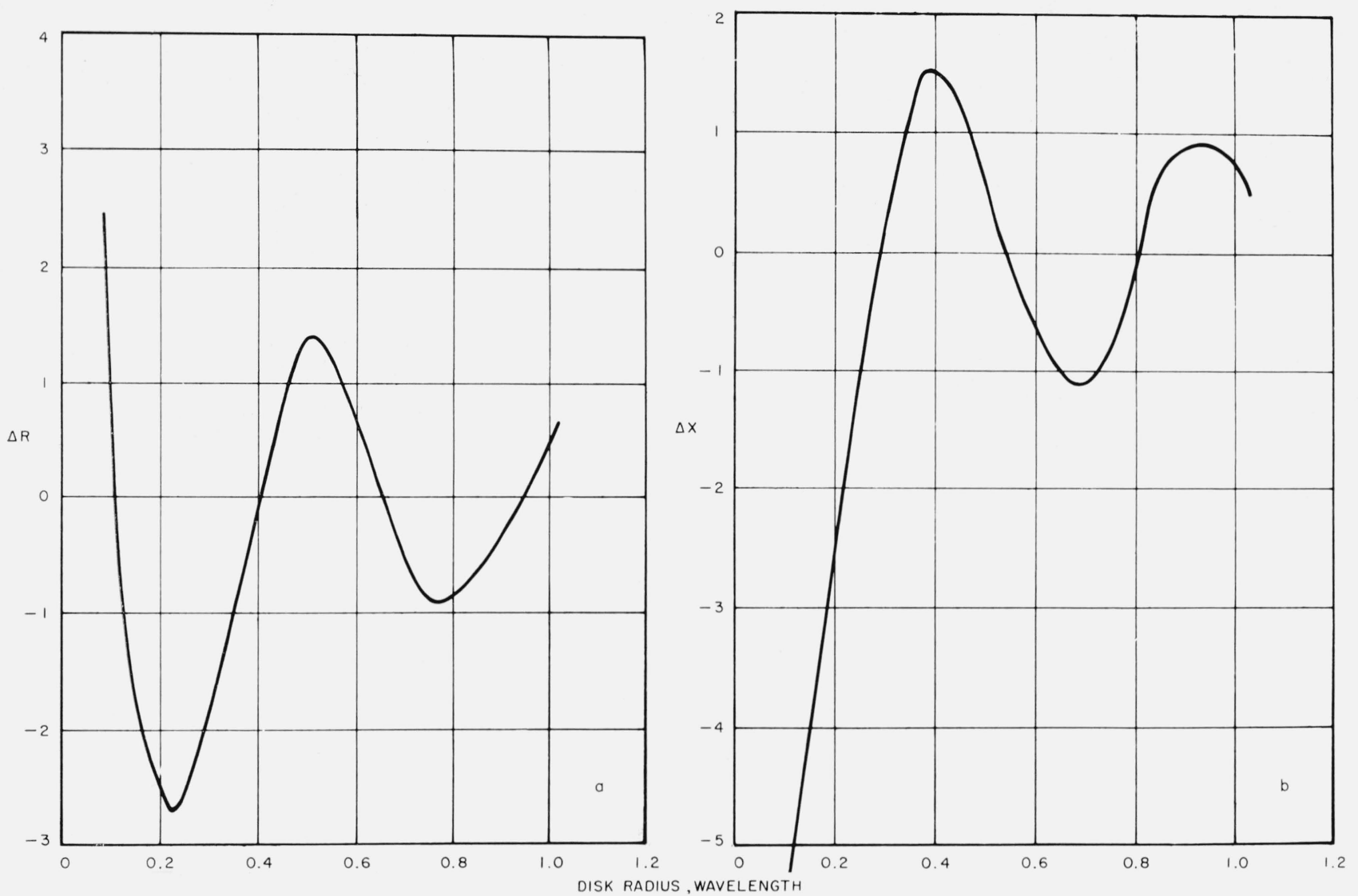

FIGURE 4. Measured values of incremental impedance, $\Delta \mathrm{Z}$, of a $\lambda / 4$ monopole with a circular conaucting disk ground system as a function of disk diameter, a.

Calculations made from the right hand side of (11) give the change, $\Delta Z$, in impedance due to the addition of the radial wires. $\Delta Z$ is zero when $N=0$. $\Delta Z$ was calculated for $\omega / 2 \pi=4.2 \mathrm{kMc} / \mathrm{s}, \quad a=0.89$, $0.242,0.299,0.366,0.433$, and 0.500 wavelengths and for $N=0,2,4,8,16$, and 32 . The imperfectly conducting ground was taken to be water for which $\epsilon_{2} / \epsilon_{0}+\sigma_{2} / i \omega \epsilon_{0}=72-i 15$. The approximation given by (4) is valid for water at the frequencies used here. The calculated values, $\Delta Z$, are plotted in figures 5 and 6 on the same charts with measured values. Calculations and measurements were also made at $9.6 \mathrm{kMc} / \mathrm{s}$, but the results were similar and are not shown here.

\section{Experimental Equipment}

The water tank was constructed of wood and was made waterproof by placing a sheet of $0.127 \mathrm{~mm}$ thick polyethylene on the bottom and sides. The tank is approximately $3.7 \mathrm{~m}$ square (50 wavelengths square at $4.2 \mathrm{kMc} / \mathrm{s}$ ) and $10 \mathrm{~cm}$ deep, and it is usually filled with water to a depth of 5 to $8 \mathrm{~cm}$ which is more than 10 skin depths at the frequencies used. ${ }^{2}$

${ }_{2}$ The skin depth of water at $4.2 \mathrm{kMc} / \mathrm{s}$ is about $4 \mathrm{~mm}$ nd at $9.6 \mathrm{kMc} / \mathrm{s}$ is about $1 \mathrm{~mm}$.
The antenna was excited by a vertical coaxial transmission line extending through the bottom of the tank. The length to diameter ratio of the monopole used was 11.2 .

The circular conducting disk ground system has been described previously [Johnk, 1960]. The radialwire ground system consists of a small disk connected to the outer conductor of the coaxial feedline. The radial wires in turn are connected to the disk and are supported at their outer ends by a structure made of methacrylate resin as shown in figure 3 . The top of the support structure is just below the water surface when in position and is sufficiently thin at the top so that its effect on the monopole impedance was negligible. Different support structures were used for different lengths of radial wires. The radial wires were bent around the support structure and then fastened to the structure several wavelengths below the water surface. It was assumed and experimentally verified that the parts of wires below the water surface had a negligible effect on the impedance.

\section{Impedance Measurements}

The impedance measurements were made by conventional slotted line techniques in the coaxial 

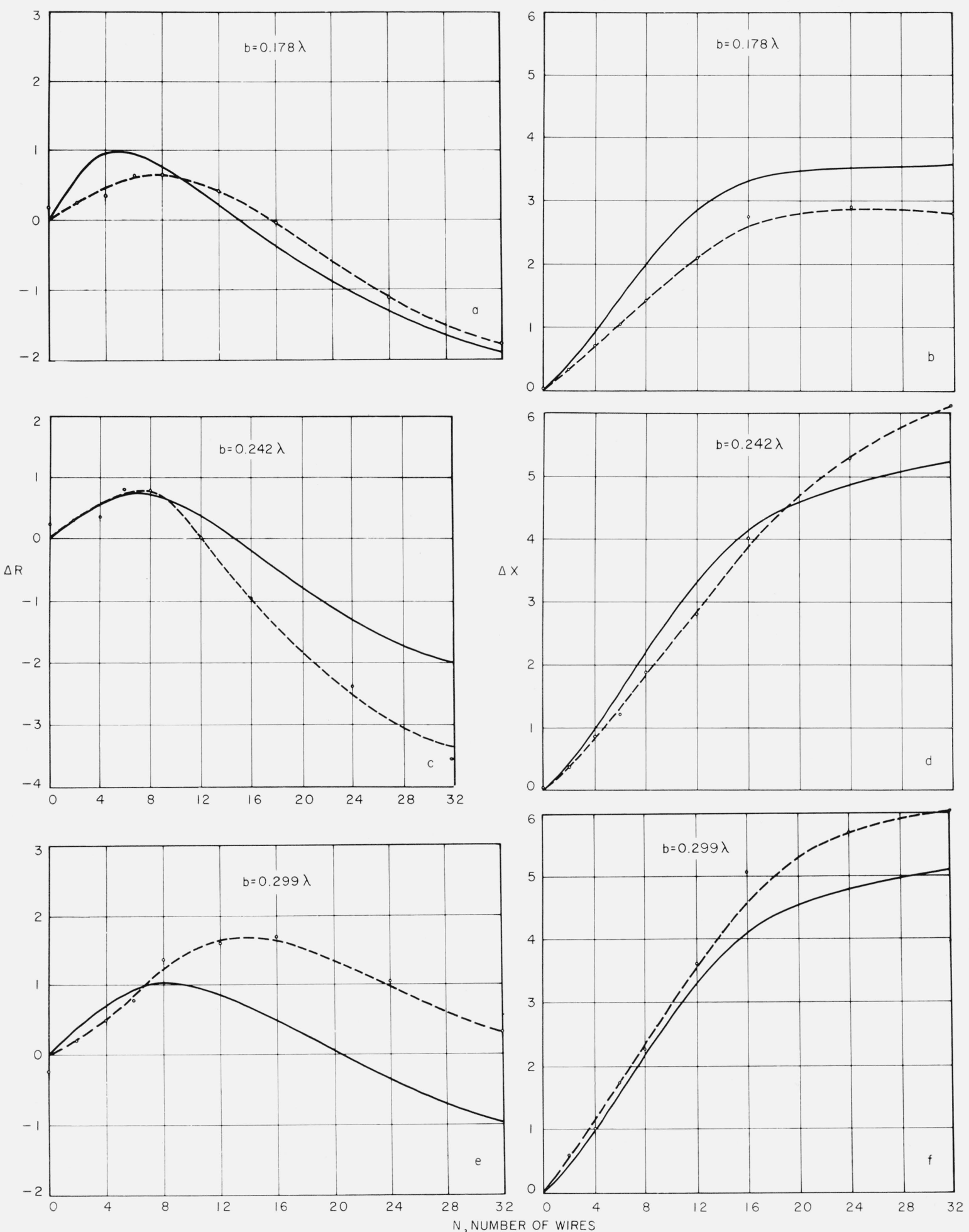

Figure 5. Measured and calculated values of change, $\Delta \mathrm{Z}$, in impedance of a $\lambda / 4$ monopole with a radial wire ground system of radius, $\mathrm{b}$, as a function of $\mathrm{N}$, the number of wires; measured values are indicated by dashed lines and calculated values by solid lines. 

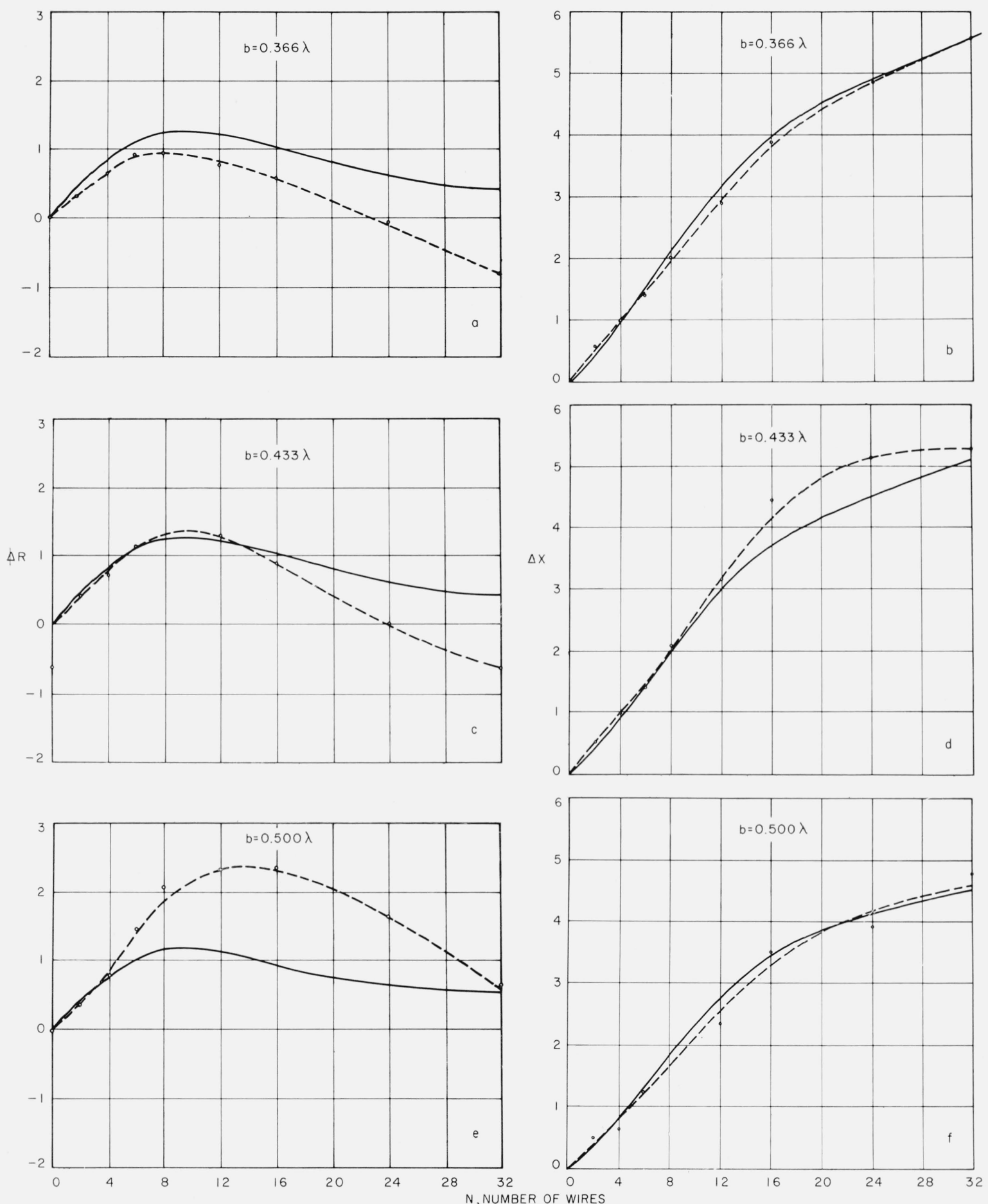

FIGURE 6. Measured and calculated values of change, $\Delta \mathrm{Z}$, in impedance of a $\lambda / 4$ monopole with a radial wire ground system of radius, $\mathrm{b}$, as a function of $\mathrm{N}$, the number of wires; measured values are indicated by dashed lines and calculated values by solid lines. 
line below the water tank. The transformation of the impedance from the slotted line to the base of the monopole has been discussed previously [Johnk, 1960].

The impedance measurements with the circular conducting disk were made by starting with a large disk and making a series of impedance measurements, machining the disk to a smaller diameter after each measurement. These measurements are shown in figure 4. Since only the derivatives of these curves are of concern in this investigation, the location of the origin for the ordinates is not of importance and was chosen such that the curves represent the change, $\Delta Z$, of impedance from that for the case of a perfectly conducting ground.

Impedance measurements were made with the radial-wire ground system by first connecting as many wires as the structure would hold and then successively measuring impedances while removing two or more radial wires after each measurement. These impedance measurements are shown in figures 5 and 6. It is the change of impedance due to the presence of the radial wires that is of primary concern in this case; therefore, the impedances in these figures are plotted as changes, $\Delta Z$, from the impedance for no radial wires. Thus $\Delta Z=0$ for $N=0$.

\section{Conclusions}

The measurements shown in figures 5 and 6 show that the effect of a radial-wire ground system on the impedance of a monopole is reasonably well predicted by (8) and (9) by Wait for the surface impedance of such a system.

The curves of incremental reactance, $\Delta X$, as a function of the number of radial wires show that $\Delta X$ increases monotonically as the number of wires is increased. This characteristic is in agreement with the calculated curves. The difference between the measured and calculated curves for $\Delta X$ is less than $1 \mathrm{ohm}$ maximum in every case; and for ground system radii of $0.366 \lambda$ and $0.500 \lambda$ it is much less.

The curves of incremental resistance, $\Delta R$, as a function of the number of wires show that $\Delta R$, for each value of ground system radii, has a maximum between $N=8$ and $N=14$. This maximum is in agreement with the calculated curves. The difference between the measured and calculated curves for $\Delta R$ is less than $1.5 \mathrm{ohms}$ in all cases.

In the derivation of (8), Wait has assumed that $\left|\gamma_{0} d\right|<<1$, where $\gamma_{0}$ is the intrinsic propagation constant in free space and $d$ is the distance between the radial wires; he further indicates that for the case in which the radial-wire system is placed on the surface of the ground the condition should be $\left|\gamma_{e} d\right|<<1$, where

$$
\gamma_{e}=\left(\frac{\gamma_{0}^{2}+\gamma^{2}}{2}\right)^{\frac{1}{2}}
$$

and $\gamma$ is the intrinsic propagation constant in the ground. The experimental measurements in figures 5 and 6 indicate that (8) gives results which agree reasonably well with measurements for values of $d$ substantially larger than the above limitations would indicate. Some of the measurements shown in figures 5 and 6 were made with $\left|\gamma_{0} d\right|$ having values of the order of 5 or higher.

Water, used as the ground in this investigation, has parameters somewhat different than usually found for an actual ground at lower frequencies. The practical problems involved in scaling an actual ground from low frequencies to microwave frequencies are substantial; therefore, rather than try to construct an actual scale model, it was decided to make measurements for a convenient ground (water) and investigate the validity of (8) and (9) for this case. Since these equations have yielded results which agree reasonably well with the measurements made in this investigation, they may be applied, with somewhat more confidence than before, to antenna systems operating at different frequencies and having grounds with different parameters. Such calculations will be presented in Part II of this paper.

\section{References}

Johnk, C. T., Impedance and pattern studies of disk-based monopoles over lossy ground surfaces, Scientific Report No. 1, ERD-TN-60-763, U. of Colo., Dept. of Elec. Engr., Contract No. AF 19(604)-4556 (July, 1960).

Maley, S. W., and R. J. King, Impedance of a monopole antenna with a circular conducting-disk ground system on the surface of a lossy half-space, J. Research NBS 65D (Radio Prop.), No. 2, 183-188 (Mar.-Apr. 1961).

Wait, J. R., and W. A. Pope, The characteristics of a vertical antenna with a radial wire ground system, App. Sci. Res. B4, $177-195$ (1954). Note that in equation (5), $\sin (\beta h-\alpha)$ should be $\sin (a-\beta h)$.

Wait, J. R., On the theory of reflection from a wire grid parallel to an interface between homogeneous media, II, Appl. Sci. Research By, 355-360 (1959). 